\title{
Hepatocyte growth factor inhibits tubular epithelial-myofibroblast transdifferentiation by suppression of angiotensin II via the JAK2/STAT3 signaling pathway
}

\author{
HONG-YUE WANG ${ }^{1}$, CHEN ZHANG $^{2}$, QING-FEI XIAO ${ }^{1}$, \\ HAI-CHUAN DOU ${ }^{1}$, YAN CHEN ${ }^{1}$, CHUN-MEI GU ${ }^{1}$ and MING-JI CUI ${ }^{1}$ \\ ${ }^{1}$ Department of Nephrology, The First Hospital of Jilin University, Changchun, Jilin 130031; \\ ${ }^{2}$ Xiamen Institute of Rare Earth Materials, Haixi Institute, Chinese Academy of Sciences, Xiamen, Fujian 361024, P.R. China
}

Received November 25, 2015; Accepted January 1, 2017

DOI: $10.3892 / \mathrm{mmr} .2017 .6301$

\begin{abstract}
Tubular epithelial-myofibroblast transdifferentiation (TEMT) is important in the development of chronic renal failure. The present study investigated whether hepatocyte growth factor (HGF) inhibits TEMT, and whether this function may be associated with the inhibition of angiotensin II (AngII) and the Janus kinase 2/signal transducer and activator of transcription 3 (JAK2/STAT3) signaling pathway. Human HK-2 kidney proximal tubular cells were divided into 4 groups and treated with AngII $\left(1 \times 10^{-6} \mathrm{M}\right), \operatorname{HGF}\left(8 \times 10^{-3} \mathrm{M}\right)$, AngII plus HGF or control conditions, followed by an assessment of apoptosis induction and the expression levels of $\alpha$-smooth muscle actin ( $\alpha$-SMA), which is a marker of TEMT. as well as the activation level of JAK2, phosphorylated (p)-JAK2, STAT3 and p-STAT3 signaling pathways. In HK-2 cells, $\alpha$-SMA mRNA and protein expression levels increased following treatment with AngII, however, decreased expression was observed following exposure to HGF. HGF counteracted the AngII-induced increase in the expression of $\alpha$-SMA in HK-2 cells. Similar expression profiles were observed for the phosphorylated forms of JAK2 and STAT3, indicating the possible involvement of this signaling pathway. The results demonstrated that treatment of cells with AngII was associated with the induction of apoptosis when compared with the control. By contrast, treatment with HGF attenuated AngII-induced apoptosis. The results suggested that HGF may inhibit TEMT by inhibiting AngII through the JAK2/STAT3 signaling pathway in HK-2 cells and HGF may prevent apoptosis induced by
\end{abstract}

Correspondence to: Professor Ming-Ji Cui or Professor Chun-Mei Gu, Department of Nephrology, The First Hospital of Jilin University, 71 Xinmin Street, Changchun, Jilin 130031, P.R. China

E-mail: why_changchun@sina.com; mingji0411@163.com

E-mail: guchunmei@163.com

Key words: hepatocyte growth factor, angiotensin II, tubular epithelial-myofibroblast transdifferentiation, Janus kinase 2/signal transducer and activator of transcription 3 signaling
AngII. The present study provides a basis for understanding the mechanisms involved in the inhibition of TEMT by HGF, which requires further investigation.

\section{Introduction}

A number of mechanisms are thought to be involved in the pathogenesis of chronic renal failure, including excessive deposition of extracellular matrix, exposure to increased cytokine levels, inhibition of apoptosis and tubular epithelial-myofibroblast transdifferentiation (TEMT) $(1,2)$. The latter process is characterized by the activation of tubular epithelial cells and their differentiation into myofibroblasts (3-5), which is considered to be the primary underlying mechanism of renal failure (6). $\alpha$-smooth muscle actin ( $\alpha$-SMA) is expressed in smooth muscle cells and myofibroblasts (7). Increased expression of $\alpha$-SMA in renal tubular epithelial cells has been suggested to be a potential marker of transdifferentiation into tubular epithelial-myofibroblasts. Hepatocyte growth factor (HGF) is a pleiotropic cytokine with multiple biological functions, including promoting karyomitosis, accelerating cell locomotion and anti-apoptotic regulation $(8,9)$. HGF is considered to possess renoprotective effects by accelerating the degradation of excessive extracellular matrix $(10,11)$, restricting TEMT and promoting hyperplasia of tubular epithelial cells (12-14).

Transforming growth factor- $\beta 1$ (TGF- $\beta 1$ ) is an important factor involved in TEMT (15). HGF is thought to antagonize the effect of TGF- $\beta 1$ by inhibiting its expression (16). In addition, angiotensin II (AngII) is thought to promote TEMT $(17,18)$. Benazepril is an angiotensin converting enzyme inhibitor that has been demonstrated to confer substantial benefits in patients with advanced renal insufficiency, particularly in those with increased urinary protein excretion $(19,20)$. In a previous study, combined treatment of HGF and benazepril demonstrated highly effective renal protection when compared to treatment with either drug alone (16). As the renoprotective effects of benazepril are mediated through inhibition of AngII expression, the authors of the present study investigated the association between HGF and AngII. It was hypothesized that TGF- $\beta 1$ repression and AngII inhibition may reduce TEMT via HGF. As renal protection by benazepril was previously 
observed to be mediated by repression of AngII expression (20), the authors investigated whether the reduction of TEMT by HGF may be mediated by AngII inhibition.

The Janus kinase 2/signal transducer and activator of transcription 3 (JAK2/STAT3) signaling pathway serves an important role in the AngII-induced proliferation of smooth muscle cells (7). A previous study demonstrated that AngII activated the JAK2/STAT3 signaling pathway and increased the expression of TGF- $\beta 1$, as well as connective tissue growth factors (21). These results indicate that AngII may contribute to renal interstitial fibrosis through the JAK2/STAT3 signaling pathway. Therefore, the association between HGF, AngII and the JAK2/STAT3 signaling pathway was investigated in the present study.

In the current study, the effect of HGF in reversing TEMT was first investigated. The association between the effects of HGF and AngII treatment, together with the potential signaling pathways involved, was subsequently examined.

\section{Materials and methods}

Materials. Human kidney proximal tubular cells (HK-2) were obtained from The Cell Bank of Type Culture Collection of Chinese Academy of Sciences (Shanghai, China). $\alpha$-SMA (cat. no. BM0002), JAK2 (cat. no. BM1219), phosphorylated (p)-JAK2 (cat. no. BA3398), STAT3 (cat. no. BA0621), p-STAT3 (cat. no. BA1709) and $\beta$-actin (cat. no. BA2305) primary antibodies were obtained from Wuhan Boster Biological Technology, Ltd. (Wuhan, China). HGF and AngII were obtained from Santa Cruz Biotechnology, Inc. (Dallas, TX, USA).

Cell culture. HK-2 cells were cultured as described previously (22). The cells were maintained in RPMI-1640 (Sigma-Aldrich; Merck Millipore, Darmstadt, Germany), and supplemented with $100 \mathrm{IU}$ penicillin, $100 \mu \mathrm{g} / \mathrm{ml}$ streptomycin (Invitrogen; Thermo Fisher Scientific, Inc., Waltham, MA, USA) and $10 \%$ fetal bovine serum (Gibco; Thermo Fisher Scientific, Inc.), in a humidified incubator at $37^{\circ} \mathrm{C}$ in $5 \% \mathrm{CO}_{2}$. Cells were divided into 4 groups and treated with AngII $\left(1 \times 10^{-6} \mathrm{M}\right)$, HGF $\left(8 \times 10^{-3} \mathrm{M}\right)$, AngII plus HGF or control conditions (RPMI-1640 with 10\% FBS) for $24 \mathrm{~h}$.

Reverse transcription-polymerase chain reaction (RT-PCR). Total RNA was isolated from cells $\left(1 \times 10^{6}\right)$ using Trizol reagent (Invitrogen; Thermo Fisher Scientific, Inc.) according to the manufacturer's instructions. First-strand cDNA was reverse transcribed using PrimeScript ${ }^{\mathrm{TM}} \mathrm{RT}$ reagent kit (Perfect Real Time; Takara Bio, Inc., Otsu, Japan). The protocol for conducting RT-PCR was identical to that described in a previous study (23). PCR products were separated by $1 \%$ agarose electrophoresis and DNA band intensities were quantified using Quantity One software (version no. 4.62; Bio-Rad Laboratories, Inc., Hercules, CA, USA). Target gene band densities were normalized to $\beta$-actin. The primers and parameters used for PCR are listed in Table I.

Western blot analysis. Cells $\left(1 \times 10^{6}\right)$ were lysed in a sodium dodecyl sulfate (SDS) sample buffer containing 2\% SDS, $10 \mathrm{mmol} / \mathrm{l}$ Tris- $\mathrm{HCl}(\mathrm{pH} 6.8)$ and $10 \%$ (v/v) glycerol. The lysates were centrifuged at $12,000 \mathrm{x} \mathrm{g}$ for $15 \mathrm{~min}$ at $4^{\circ} \mathrm{C}$, and the supernatant was stored at $-70^{\circ} \mathrm{C}$. Protein concentration was determined using a bicinchoninic acid assay kit (Bio-Rad Laboratories, Inc.). Total protein $(50 \mu \mathrm{g})$ was loaded in each lane, before it was separated in a 10\% SDS-PAGE gel and transferred to a nitrocellulose membrane. Following blocking in $4 \%$ non-fat dry milk in TBS, the membranes were incubated with primary antibodies ( $\alpha$-SMA, JAK2, p-JAK2, STAT3 or p-STAT3) at a 1:1,000 dilution in TBS overnight at $4^{\circ} \mathrm{C}$. Following washing with TBS-0.5\% Tween-20, the membranes were incubated for $1 \mathrm{~h}$ at $37^{\circ} \mathrm{C}$ with a horseradish peroxidase-conjugated anti-mouse IgG secondary antibody (cat. no. 7076; Cell Signaling Technology, Inc., Danvers, MA, USA) at 1:2,000 dilution, and immunoreactive proteins were detected using SuperSignal chemiluminescence reagent (Pierce; Thermo Fisher Scientific, Inc.). The blots were stripped and reprobed with $\beta$-actin antibody (dilution, 1:5,000). The immunoblots were analyzed by densitometry, and protein band densities were quantified using Quantity One software (version no. 4.62; Bio-Rad Laboratories, Inc.).

Acridine orangelethidium bromide staining. HK- 2 cells $\left(5 \times 10^{4}\right)$ were cultured in 24-well plates and divided into 4 groups that were treated with AngII $\left(1 \times 10^{-6} \mathrm{M}\right)$, $\mathrm{HGF}\left(8 \times 10^{-3} \mathrm{M}\right)$, AngII plus HGF or control conditions. Following incubation for $24 \mathrm{~h}$ at $37^{\circ} \mathrm{C}$ in $5 \% \mathrm{CO}_{2}, 5 \mu \mathrm{l}(10 \mu \mathrm{g} / \mathrm{ml})$ acridine orange and $5 \mu \mathrm{l}(10 \mu \mathrm{g} / \mathrm{ml})$ ethidium bromide were applied to each well, before the cells were incubated for $5 \mathrm{~min}$ at room temperature. The stained cells were analyzed using a fluorescence microscope (Olympus Corporation, Tokyo, Japan). The experiments were repeated three times.

Analysis of apoptosis by Annexin $V$ staining. In order to determine the level of apoptosis in HK-2 cells in each treatment group, Annexin V staining was performed using the Annexin V-FITC Apoptosis Detection kit (cat. no. ab14085; Abcam, Cambridge, UK) according to the manufacturer's instructions. Briefly, HK-2 cells $\left(5 \times 10^{5}\right)$ were stained with Annexin V-fluorescein isothiocyanate (FITC) and propidium iodide (PI), and incubated in the dark at room temperature for 30 min following exposure to AngII, HGF, or AngII plus HGF for $24 \mathrm{~h}$. Cells $\left(1 \times 10^{5}\right)$ were subsequently analyzed using a flow cytometer (Beckman Coulter, Inc., Brea, CA, USA) following the addition of binding buffer and the results were analyzed with Navios tetra software (version no. 1.1; Beckman Coulter, Inc.). Annexin $\mathrm{V}^{+} / \mathrm{PI}^{-}$cells were defined as cells in early apoptosis and Annexin $\mathrm{V}^{+} / \mathrm{PI}^{+}$cells were defined as cells in late apoptosis or necrosis.

Statistical analysis. Data are expressed as mean \pm standard error. One-way analysis of variance followed by the Tukey test for multiple comparisons was conducted to assess the differences among multiple groups. SPSS 17.0 (SPSS, Inc., Chicago, IL, USA) was used for data analysis. $\mathrm{P}<0.05$ was considered to indicate a statistically significant difference.

\section{Results}

$H G F$ decreases $\alpha$-SMA expression. To investigate the role of HGF and AngII in TEMT, the expression of $\alpha$-SMA in 
Table I. Primer sequences and thermal cycling conditions.

\begin{tabular}{|c|c|c|c|c|c|}
\hline Gene & Primer & Sequence $\left(5^{\prime}-3^{\prime}\right)$ & $\begin{array}{c}\text { Annealing } \\
\text { temperature }\left({ }^{\circ} \mathrm{C}\right)\end{array}$ & $\begin{array}{l}\text { Number } \\
\text { of cycles }\end{array}$ & Product (bp) \\
\hline \multirow[t]{2}{*}{$\alpha-\mathrm{SMA}$} & Sense & ACTGGGACGACTAGGAAAAA & 58 & 28 & 240 \\
\hline & Antisense & CATCTCCAGAGTCCAGCACA & & & \\
\hline \multirow[t]{2}{*}{$\beta$-actin } & Sense & ATCATGTTTGAGACCTTCAACA & 58 & 28 & 552 \\
\hline & Antisense & CATGGTGGTGCCGCCAGACAG & & & \\
\hline
\end{tabular}

$\alpha$-SMA, $\alpha$-smooth muscle actin.

A

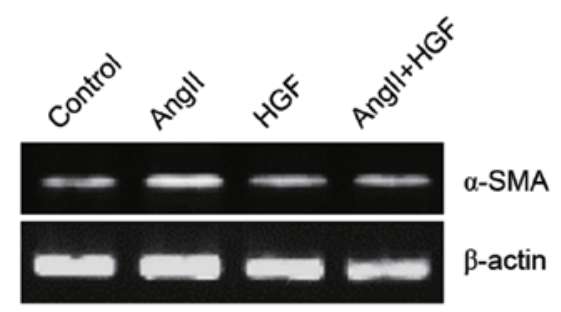

B

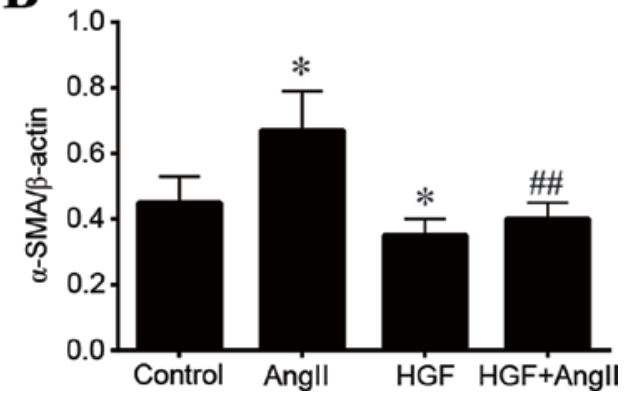

Figure 1. Regulation of $\alpha$-SMA at the mRNA level. (A) $\alpha$-SMA expression in HK-2 cells following exposure to AngII (1x10-6 M), HGF (8x10-3 M), AngII plus HGF or control conditions, as determined by reverse transcription-quantitative polymerase chain reaction analysis. (B) Quantification of $\alpha$-SMA mRNA levels relative to $\beta$-actin mRNA expression in all treatment groups by densitometry analysis. ${ }^{*} \mathrm{P}<0.05$ vs. control; ${ }^{\#} \mathrm{P}<0.01$ vs. AngII-only treated group. $\alpha$-SMA, $\alpha$-smooth muscle actin; AngII, angiotensin II; HGF, hepatocyte growth factor.

A
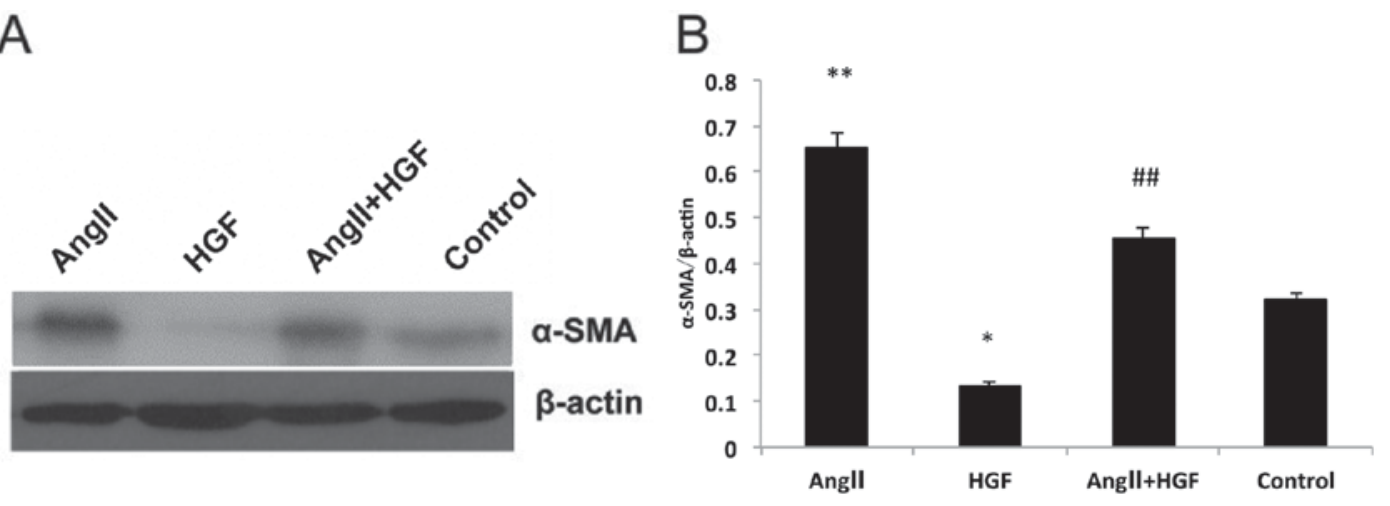

Figure 2. Regulation of $\alpha$-SMA at the protein level. (A) Representative western blot image of $\alpha$-SMA protein expression levels in HK-2 cells following exposure to AngII, HGF, AngII plus HGF or control conditions. (B) Quantification of $\alpha$-SMA protein expression relative to $\beta$-actin by densitometry analysis. * $<<0.05$ and ${ }^{* *} \mathrm{P}<0.01$ vs. control; ${ }^{\# \#} \mathrm{P}<0.01$ vs. AngII-only treated group. $\alpha$-SMA, $\alpha$-smooth muscle actin; AngII, angiotensin II; HGF, hepatocyte growth factor.

HK-2 cells was examined at the RNA and protein levels. HGF significantly decreased the expression of $\alpha$-SMA at the mRNA level when compared with the controls $(\mathrm{P}<0.05$; Fig. 1). By contrast, AngII increased $\alpha$-SMA expression when compared to control conditions $(\mathrm{P}<0.05$; Fig. 1). In addition, HGF significantly attenuated AngII-induced expression of $\alpha$-SMA mRNA $(\mathrm{P}<0.01$ vs. AngII-only treated cells; Fig. 1). A similar $\alpha$-SMA expression profile was observed at the protein level. Exposure to HGF significantly decreased $\alpha$-SMA protein expression when compared with the controls $(\mathrm{P}<0.05$; Fig. 2). However, AngII treatment significantly increased $\alpha$-SMA expression relative to that of control cells $(\mathrm{P}<0.01$; Fig. 2). In addition, exposure to HGF significantly attenuated AngII-induced increase $\alpha$-SMA expression ( $\mathrm{P}<0.01$ vs. AngII-only treated cells; Fig. 2). As $\alpha$-SMA expression is considered to provide a measure of TEMT, it is possible that AngII may promote the transdifferentiation process, whilst HGF may have the opposite effect. It is therefore possible that HGF may regulate TEMT by inhibiting AngII.

Regulation of JAK2 and p-JAK2 proteins. To investigate the association between HGF, AngII and the JAK2/STAT3 signaling pathway, the protein expression levels of JAK 2 and STAT3, as well as the phosphorylated forms of these proteins, were examined. p-JAK2 protein expression was significantly 

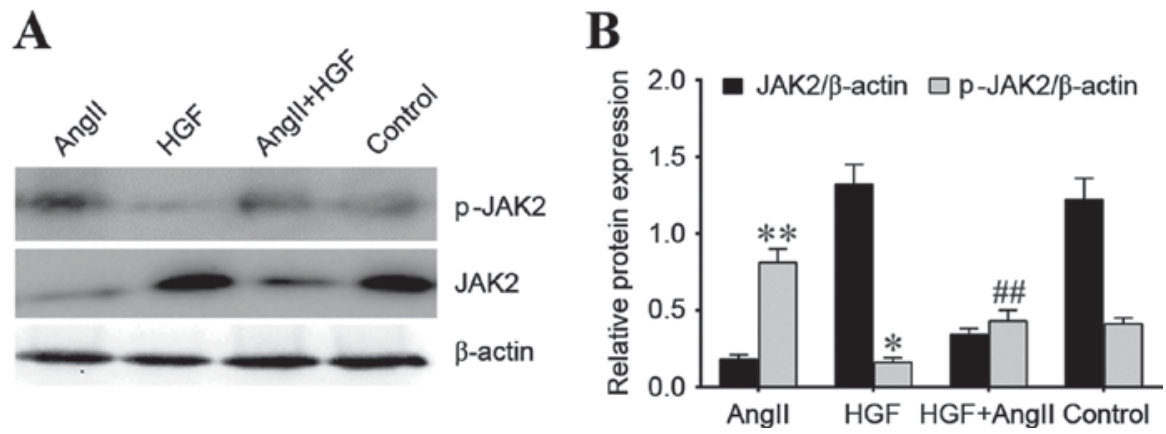

Figure 3. JAK2 and p-JAK2 protein expression levels. (A) A representative western blot image of JAK2 and p-JAK2 protein expression following exposure of HK-2 cells to AngII, HGF, AngII plus HGF or control conditions for $24 \mathrm{~h}$. (B) Quantification of JAK2 and p-JAK2 protein expression levels relative to $\beta$-actin. ${ }^{*} \mathrm{P}<0.05$ and ${ }^{* *} \mathrm{P}<0.01$ vs. control; ${ }^{\# \#} \mathrm{P}<0.01$ vs. AngII-only treated group. JAK2, Janus kinase 2; p-JAK2, phosphorylated-JAK2; AngII, angiotensin II; HGF, hepatocyte growth factor.
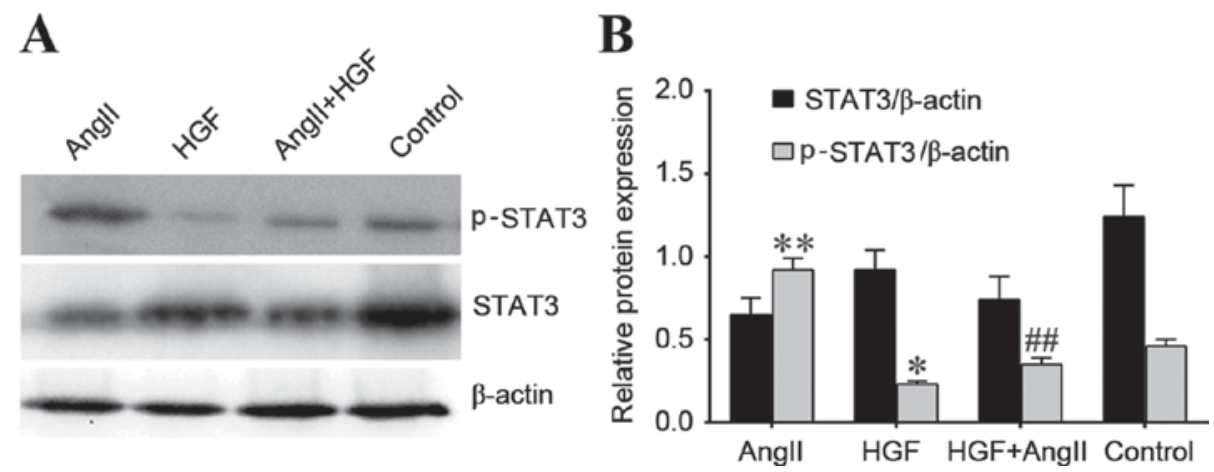

Figure 4. STAT3 and p-STAT3 protein expression. (A) A representative western blot image of STAT3 and p-STAT3 protein expression in HK-2 cells following exposure to AngII, HGF, AngII plus HGF or control conditions for $24 \mathrm{~h}$. (B) Quantification of STAT3 and p-STAT3 protein expression levels relative to $\beta$-actin. ${ }^{*} \mathrm{P}<0.05$ and ${ }^{* *} \mathrm{P}<0.01$ vs. control; ${ }^{\# \#} \mathrm{P}<0.01$ vs. AngII-only treated group. STAT3, signal transducer and activator of transcription 3 ; p-STAT3, phosphorylated-STAT3; AngII, angiotensin II; HGF, hepatocyte growth factor.

decreased in the HGF treatment group when compared with the control group $(\mathrm{P}<0.05)$, whereas $\mathrm{p}$-JAK2 protein expression was significantly increased in the AngII treatment group when compared with the controls ( $\mathrm{P}<0.01$; Fig. 3 ). p-JAK2 protein expression was significantly decreased in the AngII plus HGF treatment group when compared to the AngII-only treatment group $(\mathrm{P}<0.01$; Fig. 3). These effects were comparable to those observed with $\alpha$-SMA expression. However, a similar trend was not observed for JAK2 protein expression. JAK2 protein expression was higher in the HGF and control groups compared with the AngII and AngII plus HGF groups (Fig. 3).

Regulation of STAT3 and p-STAT3 protein expression. HGF treatment significantly decreased p-STAT3 protein expression levels when compared with controls ( $\mathrm{P}<0.05$; Fig. 4). However, AngII treatment increased p-STAT3 expression when compared with controls ( $\mathrm{P}<0.01$; Fig. 4). In addition, expression of p-STAT3 protein following exposure to AngII and HGF was significantly decreased when compared to AngII-only treated cells $(\mathrm{P}<0.01$; Fig. 4$)$. This was similar to the trend in expression of $\alpha$-SMA mRNA and protein among treatment groups. However, a similar trend to p-STAT3 was not observed for STAT3 protein expression. STAT3 protein expression was higher in the HGF and control groups compared with the AngII and AngII plus HGF groups. These results suggest that HGF may inhibit TEMT through the inhibition of AngII, and this effect may be mediated by inhibition of the p-JAK2/p-STAT3 signaling pathway.

Acridine orangelethidium bromide staining. Following exposure to AngII, HGF or AngII plus HGF, HK-2 cells were stained with acridine orange and ethidium bromide to determine the level of apoptosis in HK-2 cells exposed to different treatments. The results demonstrated that treatment of cells with AngII was associated with induction of apoptosis when compared with controls (Fig. 5). By contrast, treatment with HGF attenuated AngII-induced apoptosis (Fig. 5).

Annexin $V$ analysis. The results of the Annexin V-FITC/PI double-fluorescence staining assay revealed that AngII treatment significantly induced apoptosis in HK-2 cells when compared with controls ( $\mathrm{P}<0.01$; Fig. 6). This was demonstrated by an increase in the percentage of Annexin $\mathrm{V}^{+} / \mathrm{PI}^{-}$and Annexin $\mathrm{V}^{+} / \mathrm{PI}^{+}$subpopulations. In addition, the percentage of apoptotic cells significantly decreased following exposure to AngII plus HGF ( $\mathrm{P}<0.01$ vs. the AngII-only treated group; Fig. 6). The present study demonstrated that $15.2 \%$ of cells underwent apoptosis (Annexin $\mathrm{V}^{+} / \mathrm{PI}^{-}$) following treatment with AngII plus HGF (Fig. 6). By contrast, 28.4\% of cells underwent apoptosis following treatment with AngII alone (Fig. 6). This indicated that HGF may prevent apoptosis induced by AngII. 


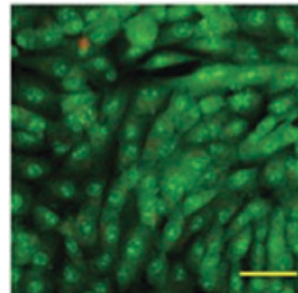

Control

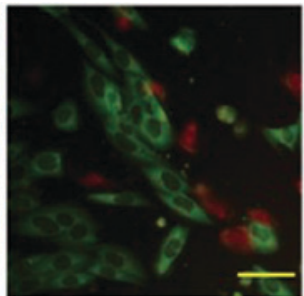

Angll

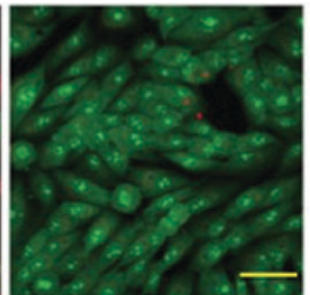

HGF

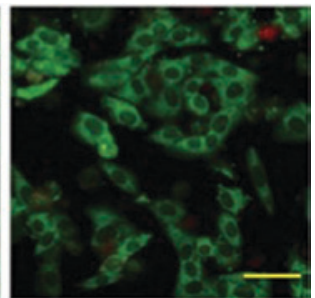

Angll+HGF

Figure 5. Analysis of apoptosis in HK-2 cells following treatment with AngII and/or HGF. HK-2 cells were exposed to AngII, HGF, AngII plus HGF or control conditions for $24 \mathrm{~h}$, and the level of apoptosis induction was assessed by acridine orange and ethidium bromide staining. Apoptotic cells are indicated by the red stain. AngII, angiotensin II; HGF, hepatocyte growth factor. (Scale bar, $200 \mu \mathrm{m}$ ).
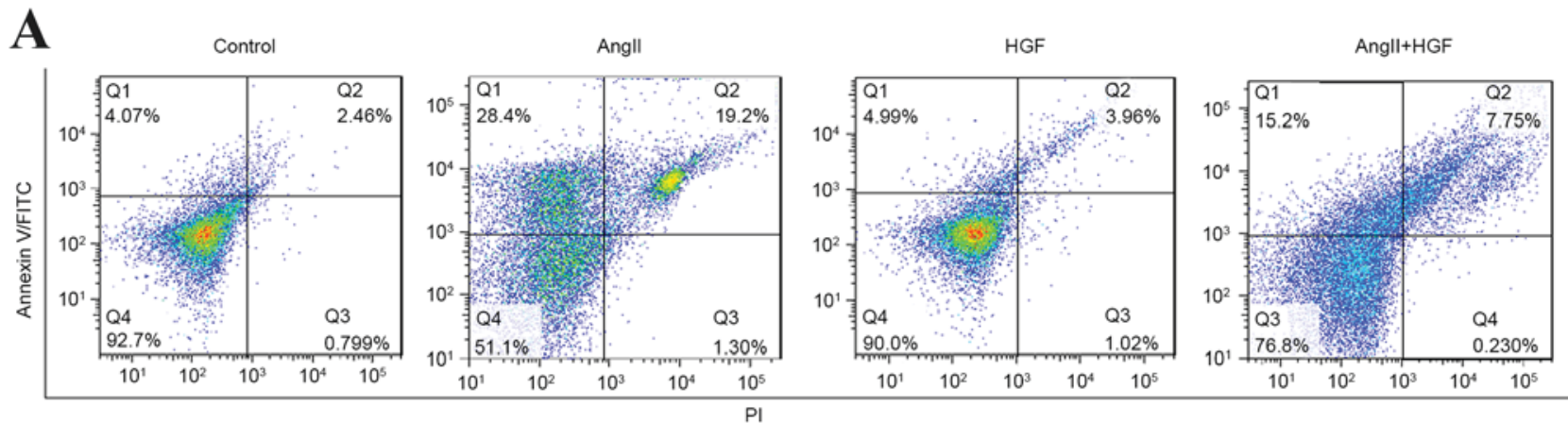

\section{B}

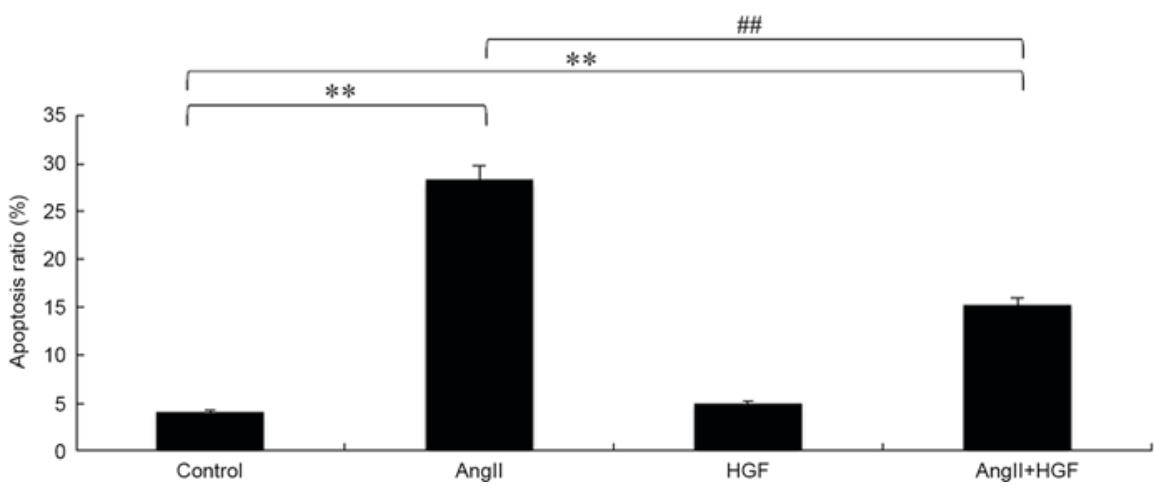

Figure 6. Induction and reduction of apoptosis in HK-2 cells by AngII and HGF, respectively. (A) HK-2 cells were exposed to AngII, HGF, AngII plus HGF or control conditions for $24 \mathrm{~h}$, and the level of apoptosis induction was assessed by Annexin V-FITC/PI staining. Apoptotic cells are indicated by the percentage of Annexin $\mathrm{V}^{+} / \mathrm{PI}{ }^{-}$cells. (B) The percentage of apoptotic HK-2 cells among treatment groups. ${ }^{* *} \mathrm{P}<0.01$ and ${ }^{\# \#} \mathrm{P}<0.01$ as indicated. AngII, angiotensin II; HGF, hepatocyte growth factor; FITC, fluorescein isothiocyanate; PI, propidium iodide.

\section{Discussion}

It has been previously demonstrated that HGF represses renal interstitial fibrosis (24-27). In addition, previous studies have revealed that HGF exhibits renoprotective effects in a number of animal models, such as acute renal failure and diabetic nephropathy models (28-30). Induction of TGF- $\beta 1$ is one of the key mechanisms responsible for increased fibrosis (31). Previous studies have indicated that HGF may induce unfavorable conditions for TEMT (10). Furthermore, increased expression of $\alpha$-SMA in the kidney has been reported to be a marker of TEMT pathology (4).

AngII is an important component of the renin-angiotensin system, and has been reported to serve an important role in a number of renal diseases (20). AngII-induced renal injury is mediated by its systemic effect on blood pressure regulation, and/or by its regulatory effect on TGF- $\beta 1(32,33)$. HGF and AngII have opposing effects, and in vascular smooth muscle cells it has been demonstrated that AngII may repress the production of HGF in a dose-dependent manner (34). Lotensin is an angiotensin converting enzyme inhibitor, that inhibits AngII production. Previous studies have demonstrated the renoprotective effect of Lotensin $(19,20)$. It is commonly used in clinical practice to decrease urinary protein excretion and to stabilize renal function during the early stages of chronic renal failure. In the present study, AngII increased $\alpha$-SMA expression at the mRNA and protein level in HK-2 cells, whereas HGF suppressed the AngII-induced expression of $\alpha$-SMA. These results indicated that, although AngII promotes TEMT, HGF may function to alleviate this process. Acridine orange/ethidium bromide staining was performed to determine functional activity. The results demonstrated that 
AngII induced apoptosis in HK-2 cells, whereas the addition of HGF was able to attenuate this effect.

The JAK2/STAT3 signaling pathway participates in the propagation of cell division, apoptosis and the regulation of immune cells, and serves an important function in diabetic kidney disease $(35,36)$. The JAK2/STAT3 signaling pathway is activated during smooth muscle cell proliferation induced by AngII. It has been suggested that the coupling of AngII with the angiotensin type 1 receptor on the surface of mesangial cells may lead to JAK2 phosphorylation, thereby binding the downstream factor, STAT3. Dimerization of STAT3 and transfer into the cell nucleus may lead to altered gene expression $(37,38)$. It was also demonstrated that AngII may increase the expression of TGF- $\beta 1$ and connective tissue growth factor by the JAK2/STAT3 signaling pathway (21). As a result of this research, the authors of the present study investigated whether AngII may be involved in the process of renal fibrosis by activating the JAK2/STAT3 signaling pathway (21). The results of the present study revealed that HGF may reduce TEMT by inhibiting AngII via by the p-JAK2/p-STAT3 signaling pathway. However, further investigation involving loss- and gain-of-function experiments, using small interfering-RNAs and/or expression vectors, are required to test this hypothesis.

\section{Acknowledgements}

The present study was supported by the Jilin Provincial Department of Health (grant no. 2009ZC041).

\section{References}

1. Yamaguchi Y, Suzuki T, Arita S, Iwashita C, Sakamoto K, Hatakeyama E, Shimmura H, Tanabe K, Ichinose M, Suzuki N and Yamada K: Possible involvement of urokinase-type plasminogen activator release from human peripheral blood lymphocytes in the pathophysiology of chronic allograft nephropathy. Transplant Proc 37: 4276-4281, 2005.

2. Wang HY, Yang LZ, Cui MJ, Gu CM, Zhao Y, Chen Y, Zhao D, Li TS and Chi B: Hepatocyte growth factor-induced amelioration in chronic renal failure is associated with reduced expression of $\alpha$-smooth muscle actin. Ren Fail 34: 862-870, 2012.

3. Grupp C, Troche I, Klass C, Köhler M and Müller GA: A novel model to study renal myofibroblast formation in vitro. Kidney Int 59: 543-553, 2001.

4. Ruiz-Ortega M, Ruperez M, Lorenzo O, Esteban V, Blanco J, Mezzano S and Egido J: Angiotensin II regulates the synthesis of proinflammatory cytokines and chemokines in the kidney. Kidney Int Suppl 82: S12-S22, 2002.

5. Okada H, Inoue T, Suzuki H, Strutz F and Neilson EG: Epithelial-mesenchymal transformation of renal tubular epithelial cells in vitro and in vivo. Nephrol Dial Transplant 15 (Suppl 6): S44-S46, 2000.

6. Badid C, Mounier N, Costa AM and Desmoulière A: Role of myofibroblasts during normal tissue repair and excessive scarring: Interest of their assesment in nephropathies. Histol Histopathol 15: 269-280, 2000.

7. Jiang T, Zhou QS, Pi L and Huang B: Role of angiotensin II and JAK2 signal pathway in transdifferentiation of renal tubular cells in mice after acute ischemic followed by reperfusion. Zhonghua Bing Li Xue Za Zhi 38: 466-471, 2009 (In Chinese).

8. Funakoshi $\mathrm{H}$ and Nakamura T: Hepatocyte growth factor: From diagnosis to clinical applications. Clin Chim Acta 327: 1-23, 2003.

9. Forte G, Minieri M, Cossa P, Antenucci D, Sala M, Gnocchi V, Fiaccavento R, Carotenuto F, De Vito P, Baldini PM, et al: Hepatocyte growth factor effects on mesenchymal stem cells: Proliferation, migration, and differentiation. Stem Cells 24: 23-33, 2006.
10. Dai $\mathrm{C}$ and Liu Y: Hepatocyte growth factor antagonizes the profibrotic action of TGF-betal in mesangial cells by stabilizing Smad transcriptional corepressor TGIF. J Am Soc Nephrol 15: 1402-1412, 2004.

11. Yang J, Dai C and Liu Y: Hepatocyte growth factor suppresses renal interstitial myofibroblast activation and intercepts Smad signal transduction. Am J Pathol 163: 621-632, 2003.

12. Li Y, Yang J, Dai C, Wu C and Liu Y: Role for integrin-linked kinase in mediating tubular epithelial to mesenchymal transition and renal interstitial fibrogenesis. J Clin Invest 112: 503-516, 2003.

13. Shimamura M, Sato N, Yoshimura S, Kaneda Y and Morishita R: HVJ-based non-viral gene transfer method: Successful gene therapy using HGF and VEGF genes in experimental ischemia. Front Biosci 11: 753-759, 2006.

14. Wang W, Li C, Summer SN, Falk S, Wang W, Ljubanovic D and Schrier RW: Role of AQP1 in endotoxemia-induced acute kidney injury. Am J Physiol Renal Physiol 294: F1473-F1480, 2008.

15. Reeves WB and Andreoli TE: Transforming growth factor beta contributes to progressive diabetic nephropathy. Proc Natl Acad Sci USA 97: 7667-7669, 2000.

16. Wang HY, Wang YJ, Cui MJ, Gu CM, Yang LZ, Zhao Y, Chen Y, Zhao D, Li TS and Chi BR: Hepatocyte growth factor-induced amelioration in renal interstitial fibrosis is associated with reduced expression of alpha-smooth muscle actin and transforming growth factor-beta1. Indian J Biochem Biophys 48: 308-315, 2011.

17. Yan Z, Yao F and Shi YH: Effects of irbesartan on expression of glycogen synthase kinase-3 $\beta$ in tubular epithelial-mesenchymal transition induced by high glucose. Chinese Pharmacological Bulletin 25: 225-229, 2009

18. Chen J, Chen JK and Harris RC: Angiotensin II induces epithelial-to-mesenchymal transition in renal epithelial cells through reactive oxygen species/Src/caveolin-mediated activation of an epidermal growth factor receptor-extracellular signal-regulated kinase signaling pathway. Mol Cell Biol 32: 981-991, 2012.

19. Ihle BU, Whitworth JA, Shahinfar S, Cnaan A, Kincaid-Smith PS and Becker GJ: Angiotensin-converting enzyme inhibition in nondiabetic progressive renal insufficiency: A controlled double-blind trial. Am J Kidney Dis 27: 489-495, 1996.

20. Hou FF, Zhang X, Zhang GH, Xie D, Chen PY, Zhang WR, Jiang JP, Liang M, Wang GB, Liu ZR and Geng RW: Efficacy and safety of benazepril for advanced chronic renal insufficiency. N Engl J Med 354: 131-140, 2006.

21. Li Y, Fan Q and Wang L: Significance of JAK2/STAT3 in angiotensin II up-regulation of TGF- $\beta 1$, CTGF and FN mRNA expression on mesangial cells under hyperglucose. J Nephrol Dialy Transplant 18: 44-48, 2009.

22. Kim YS, Xu ZG, Reddy MA, Li SL, Lanting L, Sharma K, Adler SG and Natarajan R: Novel interactions between TGF- beta 1 actions and the 12/15-lipoxygenase pathway in mesangial cells. J Am Soc Nephrol 16: 352-362, 2005.

23. Ionescu E, Sauter JF and Jeanrenaud B: Abnormal oral glucose tolerance in genetically obese (fa/fa) rats. Am J Physiol 248: E500-E506, 1985.

24. Liu Y, Rajur K, Tolbert E and Dworkin LD: Endogenous hepatocyte growth factor ameliorates chronic renal injury by activating matrix degradation pathways. Kidney Int 58: 2028-2043, 2000.

25. Mizuno S, Kurosawa T, Matsumoto K, Mizuno-Horikawa Y, Okamoto M and Nakamura T: Hepatocyte growth factor prevents renal fibrosis and dysfunction in a mouse model of chronic renal disease. J Clin Invest 101: 1827-1834, 1998.

26. Azuma H, Takahara S, Matsumoto K, Ichimaru N, Wang JD, Moriyama T, Waaga AM, Kitamura M, Otsuki Y and Okuyama A: Hepatocyte growth factor prevents the development of chronic allograft nephropathy in rats. J Am Soc Nephrol 12: 1280-1292, 2001

27. T X and Zhang L: Hepatocyte growth factor and its effect on the kidney damage. Chemistry of Life 25: 399-401, 2005.

28. Yamasaki N, Nagano T, Mori-Kudo I, Tsuchida A, Kawamura T, Seki H, Taiji M and Noguchi H: Hepatocyte growth factor protects functional and histological disorders of $\mathrm{HgCl}(2)$-induced acute renal failure mice. Nephron 90: 195-205, 2002.

29. Cruzado JM, Lloberas N, Torras J, Riera M, Fillat C, Herrero-Fresneda I, Aran JM, Alperovich G, Vidal A and Grinyó JM: Regression of advanced diabetic nephropathy by hepatocyte growth factor gene therapy in rats. Diabetes 53: 1119-1127, 2004.

30. Yang J and Liu Y: Delayed administration of hepatocyte growth factor reduces renal fibrosis in obstructive nephropathy. Am J Physiol Renal Physiol 284: F349-F357, 2003. 
31. Tsuchida K, Zhu Y, Siva S, Dunn SR and Sharma K: Role of Smad4 on TGF-beta-induced extracellular matrix stimulation in mesangial cells. Kidney Int 63: 2000-2009, 2003.

32. Rekola S, Bergstrand A and Bucht H: Deterioration rate in hypertensive IgA nephropathy: Comparison of a converting enzyme inhibitor and beta-blocking agents. Nephron 59: 57-60, 1991.

33. Taal MW and Brenner BM: Renoprotective benefits of RAS inhibition: From ACEI to angiotensin II antagonists. Kidney Int 57: 1803-1817, 2000

34. Nakano N, Morishita R, Moriguchi A, Nakamura Y, Hayashi SI Aoki M, Kida I, Matsumoto K, Nakamura T, Higaki J and Ogihara T: Negative regulation of local hepatocyte growth factor expression by angiotensin II and transforming growth factor-beta in blood vessels: Potential role of HGF in cardiovascular disease. Hypertension 32: 444-451, 1998

35. Lu TC, Wang ZH, Feng X, Chuang PY, Fang W, Shen Y, Levy DE, Xiong H, Chen N and He JC: Knockdown of Stat3 activity in vivo prevents diabetic glomerulopathy. Kidney Int 76 63-71, 2009.
36. Pang M, Ma L, Gong R, Tolbert E, Mao H, Ponnusamy M, Chin YE, Yan H, Dworkin LD and Zhuang S: A novel STAT3 inhibitor, S3I-201, attenuates renal interstitial fibroblast activation and interstitial fibrosis in obstructive nephropathy. Kidney Int 78: 257-268, 2010.

37. Levy $\mathrm{O}$ and Granot Y: Arginine-Vasopressin activates the JAK-STAT pathway in vascular smooth muscle cells. J Biol Chen 281: 15597-15604, 2006.

38. Banes AK, Shaw S, Jenkins J, Redd H, Amiri F, Pollock DM and Marrero MB: Angiotensin II blockade prevents hyperglycemia-induced activation of JAK and STAT proteins in diabetic rat kidney glomeruli. Am J Physiol Renal Physiol 286: F653-F657, 2004. 